EPJ Web of Conferences 116, 08002 (2016)

DOI: $10.1051 /$ epjconf/201611608002

(C) Owned by the authors, published by EDP Sciences, 2016

\title{
Present theoretical uncertainties on charm hadroproduction in QCD and prompt neutrino fluxes
}

\author{
M.V. Garzellia ${ }^{\mathrm{a}}$, S. Moch, and G. Sigl \\ University of Hamburg, II Institute for Theoretical Physics, 22761 Hamburg, Germany
}

\begin{abstract}
Prompt neutrino fluxes are basic backgrounds in the search of high-energy neutrinos of astrophysical origin, performed by means of full-size neutrino telescopes located at Earth, under ice or under water. Predictions for these fluxes are provided on the basis of up-to-date theoretical results for charm hadroproduction in perturbative QCD, together with a comprehensive discussion of the various sources of theoretical uncertainty affecting their computation, and a quantitative estimate of each uncertainty contribution.
\end{abstract}

\section{Introduction}

Very large volume neutrino telescopes (VLVvTs) offer the opportunity of observing high-energy neutrinos, through their interactions deep in ice or water volumes where the detector modules are installed. The IceCube collaboration, in particular, has reported evidence for the existence of a highenergy lepton flux with increasing statistics over the years [1]. At present, the best-fit to the data looks quite compatible or even suggests an astrophysical interpretation of the observed signal, but no specific correlation with particular galactic or extragalactic sources has been identified, at least so far, thus the origin of the IceCube events still remains a mystery. Many tentative hypotheses have been formulated to explain this signal [2], taking into account that neutrinos produced in a number of astrophysical environments may actually reach us after travelling long distances undeflected by cosmic magnetic fields. Possibly interesting production sites range from galactic or extragalactic sources and their neighborhood, to dark matter (DM) populated regions where heavy DM decay or DM-DM annihilation could occur. In all cases, neutrinos produced by the interaction of cosmic rays (CRs) with the Earth's atmosphere represent a background that one has to subtract in order to disentangle the signal of truly astrophysical origin. Actually, taking into account that the CR spectrum at the top of the Earth's atmosphere is peaked in the region $E_{C R} \sim 0.2-1 \mathrm{GeV}$ per nucleon for all ions, the bulk of atmospheric neutrinos, i.e., of neutrinos produced in the atmosphere, has energies $E_{v} \sim 0.05-0.4 \mathrm{GeV}$. These neutrinos are mainly the results of hadronic interactions between the impinging CRs and the light atmospheric nuclei (mostly nitrogen and oxygen), coming from intermediate charged pions and kaons, which subsequently decay leptonically. However, at increasing CR primary energy, the decay lengths of these light mesons increase, up to and exceeding the transverse size of the atmosphere. In these conditions, $\pi^{ \pm}$and $K^{ \pm}$decay probabilities are suppressed with respect to those of their

\footnotetext{
ae-mail: maria.vittoria.garzelli@desy.de
}

This is an Open Access article distributed under the terms of the Creative Commons Attribution License 4.0, which permits unrestricted use, distribution, and reproduction in any medium, provided the original work is properly cited. 

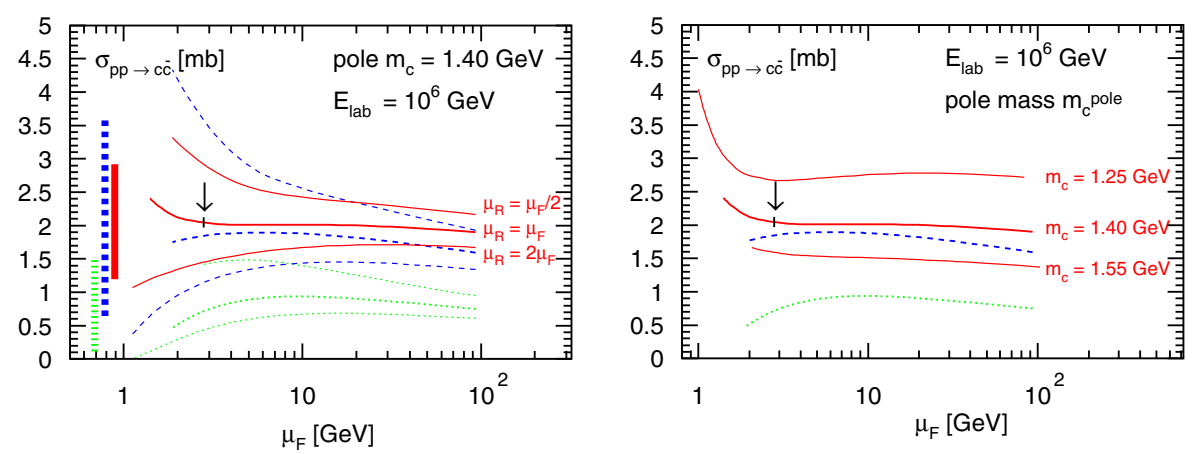

Figure 1. Left panel: cross-section for $c \bar{c}$ hadroproduction as a function of $\mu_{F}$ for different values of $\mu_{R}$. Charm mass is fixed to $m_{\text {charm }}=1.4 \mathrm{GeV}$ in the $\mathrm{OS}$ renormalization scheme. The predictions in red, blue and green refer to NNLO, NLO and LO approximations, respectively. At each fixed order, the central line refers to predictions with $\mu_{R}=\mu_{F}=m_{\text {charm }}$, whereas the upper and lower lines denotes predictions for $\mu_{R}=\mu_{F} / 2$ and $\mu_{R}=2 \mu_{F}$, respectively. Right panel: cross-section for $c \bar{c}$ hadroproduction as a function of $\mu_{F}$. $\mu_{R}$ is fixed to $\mu_{R}=\mu_{F}$. Varying the charm mass value in the range $m_{\text {charm }}=1.4 \pm 0.15 \mathrm{GeV}$ gives rise to the three NNLO predictions denoted by red lines, whereas blue and green lines corresponds to NLO and LO predictions for the central $m_{\text {charm }}$ value.

reinteraction, and v's are mainly generated by other mechanisms, especially the atmospheric production of charmed mesons and baryons, followed by their prompt decay. Charmed hadrons in fact have decay lengths smaller than $\pi^{ \pm}$and $K^{ \pm}$: the prompt neutrino flux, initiated by charm, becomes larger than the conventional one, initiated by light mesons, at an energy called "transition energy". The exact value of this energy is still subject to sizable uncertainties, mainly reflecting those affecting highly energetic hadronic interactions in the atmosphere. We have recently re-evaluated charm hadroproduction in the atmosphere [3], in the light of recent QCD theoretical and experimental progresses, triggered by the flourishing activities at hadron colliders, in particular the Large Hadron Collider (LHC): in this contribution we mainly review and further discuss the results presented more extensively in that paper.

\section{Charm hadroproduction in QCD and prompt neutrino fluxes}

In QCD the collinear factorization formalism allows the total cross-section for charm hadroproduction to be written as a convolution of a non-perturbative component, involving the parton distribution functions (PDFs) and the fragmentation functions (FF), with a perturbative part given by the partonic crosssections for the production of charm quarks from initial state quarks and gluons. In the following, we assume that the collision of a CR with an atmospheric nucleus can be simply described as a superposition of Nucleon-Nucleon $(N N)$ interactions. Recent progress in pQCD gives nowadays the possibility to calculate the total cross-section for $N N \rightarrow c \bar{c}$ pair hadroproduction including radiative corrections up to next-to-next-to-leading order (NNLO), by properly combining NNLO PDFs and NNLO partonic amplitudes. In practice, we obtained this cross-section as a function of the $N N$ collision energy by extending the HATHOR event generator [4], originally developed for $t \bar{t}$ hadroproduction. The truncation of the QCD perturbative series at a fixed order makes the theoretical predictions for the crosssections dependent of two unphysical scales: the renormalization scale $\mu_{R}$ and the factorization scale $\mu_{F}$. Unfortunately, there is no unique unambiguous way derived from first principles to properly fix these scales. We fix $\mu_{R}$ and $\mu_{F}$ by looking for minimal sensitivity, i.e. in such a way to minimize the impact of radiative corrections when comparing next-to-leading order (NLO) to NNLO predictions. The point of minimal sensitivity approximately corresponds to the choice $\mu_{F}=\mu_{R}=\mu_{0}=2 m_{\text {charm }}$, denoted by an arrow in Fig. 1a. Besides the total cross-section, we are interested in differential ones. Thus we translate 

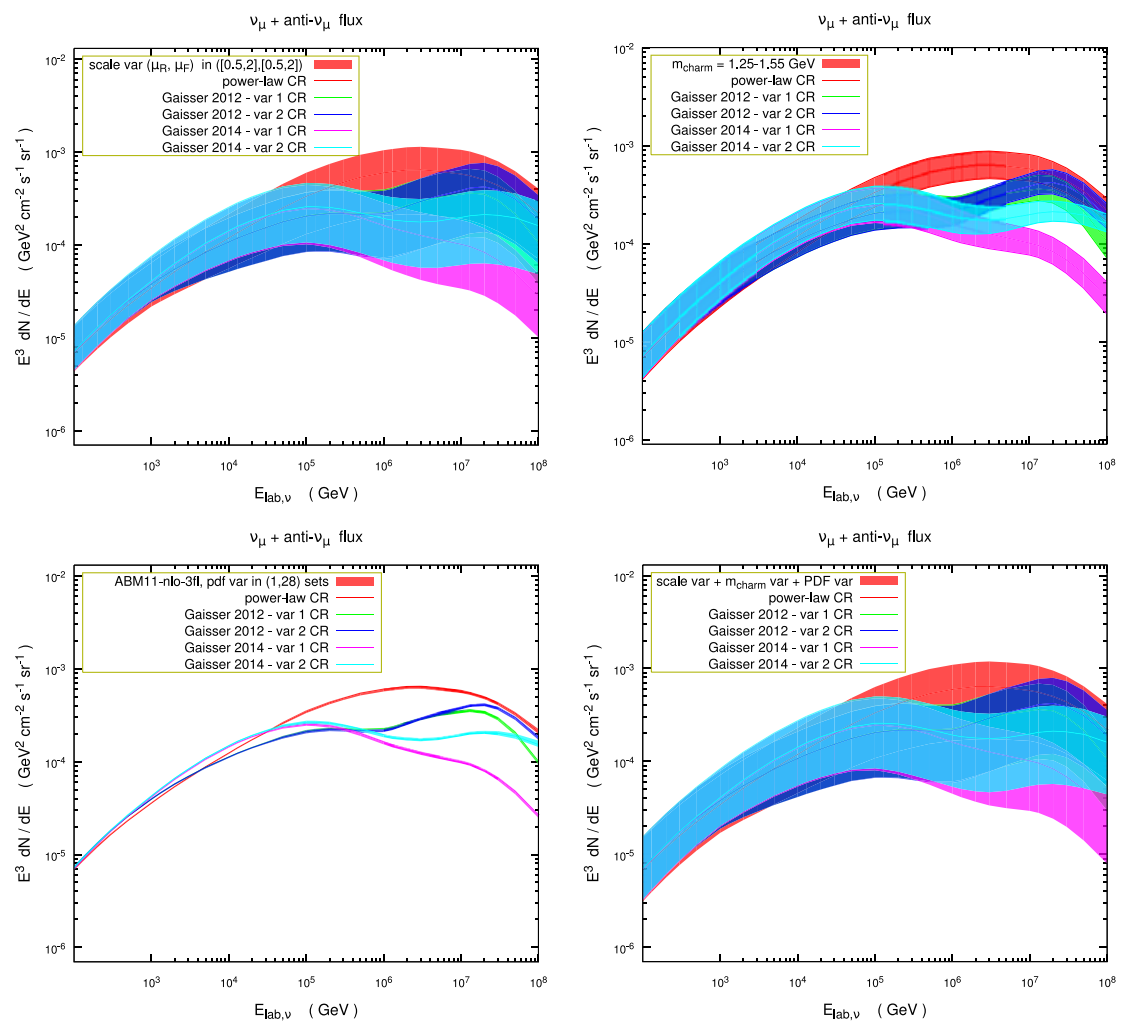

Figure 2. Prompt $\left(v_{\mu}+\bar{v}_{\mu}\right)$ fluxes as a function of neutrino energies: QCD uncertainties due to scale, mass, PDF variation and their combination in quadrature are shown in panels a, b, c, d, respectively. For each panel, predictions related to the use of different primary $\mathrm{CR}$ all-nucleon spectra are shown by different colors: the broken power-law spectrum is in red, whereas four more modern spectra (see Ref. [13] and references therein) are shown with other colors.

this static scale into a dynamical one $\mu_{0}=\sqrt{p_{T, \text { charm }}^{2}+m_{\text {charm }}^{2}}$, to better catch the kinematical aspects of charm hadroproduction. We take this as our central scale choice for the generation of differential distributions, and then we calculate scale uncertainties by considering the independent variation of $\mu_{R}$ and $\mu_{F}$ in the range $[0.5,2] \mu_{0}$, keeping out the two extreme combinations $\left(\mu_{R}, \mu_{F}\right)=(2,0.5)$ and $(0.5,2) \mu_{0}$, as prescribed in Ref. [5].

Furthermore, the $c \bar{c}$ hadroproduction cross-sections are sensitive to the precise value of charm mass, as shown in Fig. 1. Particular care has to be taken in the choice of this parameter especially when adopting the on-shell (OS) scheme to renormalize heavy-quark masses, because this scheme shows a poor perturbative convergence, actually worse than the modified minimal subtraction $(\overline{M S})$ scheme [6]. We fix the charm mass value to $m_{\text {charm }}^{O S}=1.40 \pm 0.15 \mathrm{GeV}$. This way the cross-section in the OS scheme approximately reproduces that in the $\overline{M S}$ scheme. We notice that the accuracy on the pole mass is also limited by the renormalon ambiguity.

Further QCD uncertainties are related to the PDF choice. At present, many uncertainties still exist at low Bjorken $x\left(x<10^{-4}\right)$, due to lack of data. However this region is progressively important when going to higher collision energies, which is a crucial issue for astrophysics because CRs in the tail of the CR energy spectrum are even more energetic than the beams at colliders. Recently, LHCb data on charm and bottom hadroproduction in mid-peripheral collisions have been used to perform PDF fits down to 
lower values of $x\left(x \sim 10^{-6}\right)$. The first fit in this direction was proposed by the PROSA collaboration in Ref. [7], followed by Ref. [8] applying the same concept to the NNPDF PDFs. In this paper we use the ABM11 PDFs [9]. We note that, although these PDFs do not take into account any LHCb data, their extrapolation in the range $10^{-6}<\times<10^{-4}$ turns out to lie within the PROSA PDF uncertainty band. We evaluate PDF uncertainties by considering the 28 ABM11 variations, accompanying the central fit available in the LHAPDF 6.1.5 interface.

Neutrino fluxes were computed by solving a system of coupled differential equations regulating particle evolution in the atmosphere, by using the $Z$-moment approach [10]. Inputs to the $Z$-moments are differential cross-sections for charmed hadron hadroproduction. Unfortunately, these distributions are not yet available at NNLO. We thus computed them through a NLO QCD + parton shower + hadronization approach, as provided by POWHEGBOX [11] + PYTHIA [12]. Charm mass, PDF and scale input of the computation and their intervals of variation were fixed on the basis of NNLO information, as explained above. The effect of uncertainties due to scale, charm mass and PDF variation on $\left(v_{\mu}+\bar{v}_{\mu}\right)$ prompt fluxes is shown in Fig. 2a, b, c, respectively. In the last panel, Fig. 2d, the combination of those QCD effects, summed in quadrature, is also shown. The total contribution of additional uncertainties related to hadronization and multiple particle interactions amounts to several ten percent. We observe that, among all QCD uncertainties, the one due to scale variation dominates. We also notice that at energies above the $\mathrm{PeV}$ scale, the contribution of astrophysical uncertainties, related to the unknown composition of the CR spectrum at primary energies above the knee, becomes progressively larger and ends up in dominating over the contribution from QCD uncertainties at ultra-high neutrino energies. The region around and above the PeV will be better probed by upgraded VLV $\nu$ Ts, like the IceCube-Gen2 project [14], providing an increase of the instrumented volume from $\sim 1 \mathrm{~km}^{3}$ to $\sim 10 \mathrm{~km}^{3}$. By the time they will start data taking, it would be advisable to reduce all uncertainties (both those from QCD and those from astrophysics) affecting the computation of prompt neutrino fluxes at high energies. Our most up-to-date predictions are available at http://promptfluxes.desy.de.

\section{References}

[1] IceCube Collaboration (M. G. Aartsen, et al.) Science 342, 1242856 (2013) [arXiv: 1311. 5238]

[2] C.S. Fong, H. Minakata, B. Panes, JHEP 1502, 189 (2015) [arXiv:1411.5318]

[3] M.V. Garzelli, S. Moch, G. Sigl, JHEP 1510, 115 (2015) [arXiv: 1507.01570]

[4] M. Aliev, H. Lacker, U. Langenfeld et al. Comput. Phys. Commun. 182, 1034 (2011) [arXiv: 1007.1327]

[5] M. Cacciari, S. Frixione, N. Houdeau et al. JHEP 1210, 137 (2012) [arXiv: 1205. 6344]

[6] S. Narison, Phys. Lett. B 197, 405 (1987)

[7] PROSA collaboration (A. Zenaiev et al.), EPJC 75, 396 (2015) [arXiv: 1503. 04581]

[8] R. Gauld, J. Rojo, L. Rottoli, J. Talbert, JHEP 1511, 009 (2015) [arXiv : 1506 . 08025]

[9] S. Alekhin, J. Blümlein, S. Moch, Phys. Rev. D 86, 054009 (2012) [arXiv : 1202 . 2281]

[10] P. Lipari, Astropart. Phys. 1, 195 (1993)

[11] S. Alioli, P. Nason, C. Oleari, E. Re, JHEP 1006, 043 (2010) [arXiv: 1002 . 2581]

[12] T. Sjostrand, S. Mrenna, P.Z. Skands, JHEP 0605, 026 (2006) [hep-ph/0603175]

[13] T. Gaisser, T. Stanev, S. Tilav, Front. Phys. China 8, 748 (2013) [arXiv: 1303. 3565]

[14] IceCube Collaboration (M. G. Aartsen, et al.) [arXiv: 1412.5106] 